\title{
Palatal brushing for the treatment of denture stomatitis: A multicentre randomized controlled trial
}

\author{
Raphael de Souza a , Carolina Chaves ${ }^{b}$, Kimia Rohani a, Sabrina Bouferguene a, Jean Barbeau b, \\ Eduardo Borie c, Benjamin Weber c, Ramon Fuentes c, Luciana Crizostomo d, \\ Claudia Silva-Lovato ${ }^{d}$, Elham Emami ${ }^{a},{ }^{*}$ \\ a Faculty of Dental Medicine and Oral Health Sciences, McGill University, Montreal, Canada \\ ${ }^{b}$ Faculté de Médecine Dentaire, Université de Montréal, Montreal, Canada \\ ' CICO Research Centre, Integral Dentistry of Adults Department, Faculty of Dentistry, Universidad de la Frontera, Temuco, Chile \\ ${ }^{d}$ Department of Dental Materials and Prostheses, School of Dentistry of Ribeirao Preto, University of Sao Paulo, Ribeirao Preto, Brazil
}

\begin{abstract}
Purpose: To determine the effectiveness of palatal brushing in the treatment of denture-related erythematous stomatitis (DES) in complete denture wearers.

Methods: This two-parallel-arm RCT was conducted in three university clinics in Brazil, Canada, and Chile. Participants $(n=77)$ were randomly allocated to receive (i) instructions for palatal brushing and standard oral/denture hygiene ("intervention"); or (ii) standard oral/denture hygiene instructions only ("control"). Data collection was carried out at the baseline and at 3 and 6 months after intervention. Outcomes included the magnitude of oral Candida carriage and the degree of inflammation of denture-bearing tissues. Groups were compared using generalized estimating equations and chi-square test $(a=0.05)$.

Results: Palatal inflammation levels were reduced significantly in the "intervention" compared to "control" group at 6 months (intervention: $70 \%$, control: $40 \%$; chi-square, $\mathrm{p}=0.04$ ). There was no between-group significant difference in the Candida count from denture and palatal biofilms; however, a subgroup analysis restricted to baseline Candida carriers showed further reduction with the intervention at 6 months. No adversity was observed by trialist or reported by participants.

Conclusion: Including palatal brushing in oral instructions for denture wearers has positive impact on DES-related mucosal inflammation. Thus, our findings endorse the inclusion of palatal brushing in standard oral hygiene instructions to treat DES.
\end{abstract}

Keywords: Denture stomatitis; Complete denture; Candida; Oral candidosis; Pragmatic trials

Received 6 October 2021, Accepted 3 February 2022, Available online 8 March 2022

\section{Introduction}

Denture-related erythematous stomatitis (DES) is a chronic inflammatory lesion of the oral mucosa covered by a removable denture, mostly found in the palate[1,2]. This condition is known to affect $15-70 \%$ of denture wearers and seems more common in elderly women, although it may also appear in children and adults wearing orthodontic appliances and obturator prosthesis[1-5]. In a large population-based study with 17,235 participants, oral mucosal inflammation conditions such as DES have been associated with an increased risk of cardiovascular diseases, and a significant risk factor for aspiration pneumonia in the frail elderly[2,6,7].

DOI: https://doi.org/10.2186/jpr.JPR_D_21_00258

*Corresponding author: Elham Emami, Faculty of Dental Medicine and Oral Health Sciences, McGill University, 2001 McGill College Ave, suite 500, H3A 1G1, Montreal, (QC) Canada.

E-mail address: elham.emami@mcgill.ca

Copyright: @ 2022 Japan Prosthodontic Society. All rights reserved.
Antifungal medication is the current treatment for DES, although scientific evidence acknowledges the high rate of rapid recurrence after the cessation of antifungals[2,8]. Prescription of antifungal medications relies on the hypothesis that Candida infection is the main etiological factor of DES[1,8]. However, previous systematic reviews and meta-analyses have shown only a limited or null effect of antifungals in DES care management[2,8]. Furthermore, resistance to antifungals and severe adverse effects such as gastrointestinal disturbances, liver and kidney toxicity, and hypersensitivity have been reported[1,9].

On the other hand, according to the literature denture hygiene is a critical etiological risk factor for DES[1]. Poor denture cleaning creates an anaerobic environment with low $\mathrm{pH}$, and leads to an increase in palatal and denture biofilm pathogens[1]. The toxins and metabolic wastes produced by these microorganisms foster DES onset and exacerbation of mucosal inflammation[1,9]. The inflammation may also result from the repeated mucosal trauma of ill-fitting prostheses[1,9,10]. The inflammation favours colonization by Candida 
species, meaning that fungal infection is not necessarily a causative factor of DES and it is mainly a moderator[1]. In fact, previous studies have demonstrated the efficacy of various hygienic approaches in preventing DES and decreasing the Candida carriage[1,8,9,11-13]. The systematic reviews and meta-analyses on this topic have shown a lower risk of DES recurrence and no outcome difference between hygiene instructions and antifungal treatment in patients suffering from this disease[8,11].

A recent single-arm trial conducted by our team has demonstrated promising results of palatal brushing, a simple and cost-effective procedure, in treating DES[14]. In that study, palatal brushing was used to mechanically remove palatal biofilm and stimulate mucosal circulation and salivary flow[14], and showed a significant reduction of palatal inflammation and Candida carriage over a period of 3 months[14]. However, these results should be validated by a randomized clinical trial. Therefore, the primary objective of this trial was to determine if palatal brushing decreases the number of Candida colonies on the palatal mucosa and denture of patients with DES. The secondary objective was to demonstrate the efficacy of palatal brushing in reducing the palatal inflammation. We hypothesized that edentulous patients who brush their palate and follow standard hygiene measures will have less denture and palatal Candi$d a$ colonies and less palatal inflammation than those using standard hygiene over a 6-month period.

\section{Methods and Materials}

\subsection{Study design and setting}

This pragmatic, multicentre randomized controlled trial was conducted in three university-based clinics in Brazil, Canada, and Chile. The study was designed as a two-arm, parallel-group trial with 1:1 allocation of participants. Institutional review board approval was obtained from each participating institution: A06-M25-16A (McGill University, Canada - coordinating centre), 16-003-CERES-D (Université de Montréal, Canada - clinical site 1), 37033814.60000 .54190 (University of São Paulo, Brazil - clinical site 2), and 013/15 (Universidad de la Frontera, Chile - clinical site 3). The protocol trial was registered in ClinicalTrials.gov (\#NCT02686632) and published previously[15].

\subsection{Study participants}

The recruitment of study participants was carried out in 20152017 via advertising in local newspapers and distributing informative brochures in dental clinics and long-term care facilities. Research project managers at each study centre called interested individuals, explained the study to them, and assessed them based on primary eligibility criteria. Potential participants were then asked to visit one of the clinical sites, where they were provided with more information related to the study and were carefully re-assessed based on the study's full eligibility criteria.

Potential participants were included if they were (i) older than 18 years; (ii) fully edentulous; (iii) regularly wearing maxillary complete conventional dentures; and (iv) presenting clinical signs compatible with a DES diagnosis. Newton class II and III were not required for inclusion, as in the original protocol[15] (reason for change: to enable adequate study enrollment rate and representability of patients in the Brazilian centre). Exclusion criteria were: (i) having an appointment to replace any existing denture (upper or lower) during the course of the study; (ii) taking antibiotics, steroids, or antifungal agents within 4 weeks prior to the initiation of the interventions; (iii) systemic conditions predisposing patients to Candida infection such as uncontrolled diabetes and history of radiotherapy or chemotherapy; (iv) other oral lesions apart from DES, including patients with other types of oral Candida infection (e.g., acute pseudomembranous candidiasis). Those who satisfied the eligibility criteria were asked to provide informed consent prior to initiation of the study and underwent complete clinical examinations, during which the baseline data was collected.

\subsection{Intervention}

Participants were randomized to either intervention or control group, with both groups receiving standard instructions on oral/ denture hygiene measures. These standard instructions were provided verbally by a researcher (with the provision of an explanatory pamphlet) as follows:

i) Washing all the internal and external surfaces of the denture with tap water to remove all visible debris;

ii) Brushing all surfaces of the denture using a denture brush (OralB Denture Brush; Procter \& Gamble) and 2-cm toothpaste for approximately two minutes;

iii) Rinsing the denture with tap water until visibly clean.

Exclusive instructions for the intervention included brushing the palate without toothpaste. A research trainee (not involved in the provision of standard instructions) provided a soft bristle manual toothbrush (Oral-B CrossAction Pro Health; Procter \& Gamble, lowa, IA, USA) to intervention group participants, and instructed them to perform palatal brushing with vertical, horizontal, and vibration movements, for approximately one minute. Participant in the intervention group also received written instructions of palatal brushing, besides standard instructions.

All participants were asked to follow the given instructions after each meal and before going to sleep, for 6 months. Data collection was conducted at three time points, including baseline (T0), first follow-up at 3 months (T2), and second follow-up (T3) at 6 months postintervention. Study participants were asked to stop palatal brushing in case of any adverse effects.

\subsection{Outcomes}

\subsubsection{Primary outcome}

The primary outcome was the magnitude of oral Candida carriage defined by Candida spp. colony forming unit (CFU) counts, originating from both denture biofilm and palatal mucosa. Biofilm specimens from dentures were taken using standardized sonication technique[16,17], and palatal biofilm specimens were collected with a sterile swab, which was put on the central part of the palate $\left(1 \mathrm{~cm}^{2}\right)$ [18], then placed in a sterile tube with $5 \mathrm{ml}$ normal saline for subsequent sonication ( $2 \mathrm{~min}$ ). All biological samples were collected between 9 a.m. and 12 p.m. to minimize circadian variation in microbial counts $[19,20]$ and were immediately sent to microbiological laboratories at each study centre. Sonicates from palate and the denture were serially diluted in saline $\left(10^{-1}\right.$ to $\left.10^{-4}\right)$ and inoculated on Sabouraud dextrose $4 \%$ agar (Difco, Becton Dickinson, Sparks, MD, USA) then incubated for 48 hours at $37^{\circ} \mathrm{C}$. CFU/ml in the original sonicate $\left(10^{\circ}\right)$ was measured after counting CFUs from the dilutions that yielded 1-300 colonies[21]. 
Table 1. Sociodemographic characteristics of study participants

\begin{tabular}{|c|c|c|c|}
\hline $\begin{array}{l}\text { Variables } \\
\text { n (\%) or Mean (SD:) }\end{array}$ & Palatal Brushing (40) & Standard Hygiene (37) & Total (77) \\
\hline \multicolumn{4}{|l|}{ Demographic } \\
\hline Age & 70 (SD 9) & 67 (SD 13) & 69 (SD 11) \\
\hline Men: Women (\%men) & $3: 33(8)$ & $9: 24(27)$ & $12: 57(17)$ \\
\hline \multicolumn{4}{|l|}{ Education level } \\
\hline • High school or less & $32(89)$ & $31(94)$ & $63(91)$ \\
\hline - College and higher & $4(11)$ & $2(6)$ & $6(9)$ \\
\hline \multicolumn{4}{|l|}{ Yearly income } \\
\hline$\cdot<\$ 10,000$ & $9(26)$ & $14(44)$ & $23(34)$ \\
\hline • $\$ 10,000-\$ 29,999$ & $22(63)$ & $15(47)$ & $37(55)$ \\
\hline$\cdot>\$ 30,000$ & $4(11)$ & $3(9)$ & $7(11)$ \\
\hline Number of past or current smokers & $19(53)$ & $15(46)$ & $34(49)$ \\
\hline Mean pack/year for past or current smoker & 25 (SD 23) & 21 (SD 21) & $23(S D 22)$ \\
\hline \multicolumn{4}{|l|}{ Alcohol consumption } \\
\hline •Never & $12(33)$ & $12(39)$ & $24(36)$ \\
\hline - Rarely & $9(25)$ & $6(19)$ & $15(22)$ \\
\hline - Occasionally & $14(39)$ & $12(39)$ & $26(39)$ \\
\hline - Frequently & $1(3)$ & $1(3)$ & $2(3)$ \\
\hline
\end{tabular}

\subsubsection{Secondary outcomes}

The secondary outcomes consisted of the degree of inflammation of denture-bearing tissues, as observed by a clinician. We classified clinical findings according to three classifications, i.e., Modified Newton[14,22], Schwartz[23], and Budtz-Jorgensen[24] classification (Supplementary Table 1). In brief, the modified Newton classification further divides class I into IA (petechiae only) and IB (localized inflamed areas), whereas classes II (generalized area of inflammation) and III (inflammation plus palatal mucosal hyperplastia) remains the same of the original classification.

The Schwartz index was used to grade the area and severity of inflammation, with each aspect based on a 4-point Likert scale (range: 0 to 3 each). Summing the area and severity scores yielded a total score ranging from 0 (healthy mucosa) to 6 (severe and widespread inflammation). Both Newton and Schwartz indices were applied on baseline and during the 3- and 6-month follow-ups. Finally, changes in inflammation over time/treatment effect were classified according to Budtz-Jorgensen, based on the difference between preand post-treatment total inflammation scores. Four categories were possible: (i) inflammation resolved/large effect; (ii) inflammation reduced/moderate effect; (iii) no change in inflammation/no effect; and (iv) increased inflammation/negative effect.

The clinicians' diagnostic calibration was conducted with an interobserver agreement of good to excellent ( $k=0.6$ to 0.84 ). Photographs of the palatal mucosa were also taken using a Nikon D90 Camera (105 mm f/2.8 D, macro flash SB-21; Nikon Co., Tokyo, Japan) to ensure diagnostic agreement among clinical investigators. The first author was present at the three clinical sites to train data collectors regarding how to classify palatal inflammation and exploratory variables, before commencing the trial. Those visits included the explanation of trial procedures with representative photos from our previous single-arm study[14], as well as demonstrations with patients that would match the eligibility criteria of this trial. All sites were visited again at least once to ensure good/similar practices, when around $50 \%$ of planned recruitment was achieved.

Participants were also asked whether they were experiencing mild pain on each time point, as a potential adverse effect of palatal brushing[14]. Answers were provided on a 4-point Likert scale (0: never; 1: rarely; 2: sometimes; 3: often).

\subsubsection{Explanatory variables}

At baseline, medical and dental history, sociodemographic, lifestyle, as well as oral health and denture-related data were collected by administrating a self-reported questionnaire and performing clinical examinations[15]. In each country, self-reported questionnaires were written in their respective official languages, i.e., French and English for site 1, Portuguese for site 2, and Spanish for site 3. Please refer to Appendix for a copy of data collection forms and detailed information on exploratory variables.

\subsection{Randomization, blinding, and allocation}

Off-site computer-assisted, centralized block randomization was used for this trial, with stratification done according to each centre. Each participant received a sequentially numbered, sealed, opaque, and tamper-proof envelope from the trial coordinator at the respective centre, containing the concealed allocated intervention.

All clinicians and those in charge of data analysis were blind to group assignment. However, the nature of the intervention did not allow for blinding the participants and the research trainee responsible for palatal brushing instructions. To minimize bias from these sources, participants were scheduled so as to avoid waiting time together, and thus avoid potential contamination. Also, the research trainee opened the envelope for each participant immediately before hygiene instructions, in the absence of other team members. 
Table 2. Medical and dental history of study participants

\begin{tabular}{|c|c|c|c|}
\hline $\begin{array}{l}\text { Variables } \\
\text { n (\%) or Mean (SD:) }\end{array}$ & Palatal Brushing (40) & Standard Hygiene (37) & Total (77) \\
\hline \multicolumn{4}{|l|}{ Medical history } \\
\hline Presence of systemic disease & $20(56)$ & $17(46)$ & $37(54)$ \\
\hline Medication use & $24(60)$ & $26(70)$ & $50(73)$ \\
\hline Immunosuppressant/Corticosteroid & $2(6)$ & $2(6)$ & $4(6)$ \\
\hline \multicolumn{4}{|l|}{ Dental history and habits } \\
\hline \multicolumn{4}{|l|}{ Consultation of oral health professional } \\
\hline$\cdot$ Never & $6(17)$ & $3(9)$ & $9(13)$ \\
\hline • Emergency & $16(44)$ & $22(67)$ & $38(55)$ \\
\hline - Once a year & $8(22)$ & $3(9)$ & $11(16)$ \\
\hline - Once or more a year & $6(17)$ & $5(15)$ & $11(16)$ \\
\hline Received instruction for denture hygiene & $15(43)$ & $15(47)$ & $30(45)$ \\
\hline Denture cleaner & $6(17)$ & $4(13)$ & $10(15)$ \\
\hline Mouthwash & $15(43)$ & $9(28)$ & $24(36)$ \\
\hline Adhesive for upper denture & $1(3)$ & $3(9)$ & $4(6)$ \\
\hline Difficulty cleaning the denture & $3(9)$ & $6(19)$ & $9(13)$ \\
\hline \multicolumn{4}{|l|}{ Food under the denture } \\
\hline • Rarely & $5(14)$ & $6(19)$ & $11(17)$ \\
\hline - Occasionally & $14(40)$ & $7(22)$ & $21(31)$ \\
\hline • Frequently & $9(26)$ & $9(28)$ & $18(27)$ \\
\hline • Very often & $7(20)$ & $10(31)$ & $17(25)$ \\
\hline Nocturnal wear (upper denture) & $19(56)$ & $13(41)$ & $32(49)$ \\
\hline \multicolumn{4}{|l|}{ Oral clinical exam } \\
\hline Mean years of edentulism & 32 (SD 15) & 27 (SD 15) & 30 (SD 15) \\
\hline Mean age of current upper prosthesis (year) & 19 (SD 16) & 14 (SD 13) & 17 (SD15) \\
\hline Number of upper prostheses & 2 (SD 3) & 2 (SD 1) & $2(\mathrm{SD} 2)$ \\
\hline Number of lower prostheses & 2 (SD 1) & 1 (SD 1) & 1 (SD 1) \\
\hline Upper denture satisfaction (0-100) & 50 (SD 32) & 50 (SD 37) & 50 (SD 34) \\
\hline Cheilitis & $2(5)$ & $2(5)$ & $4(5)$ \\
\hline \multicolumn{4}{|l|}{ Saliva quality } \\
\hline • Normal & $31(80)$ & $32(87)$ & $63(83)$ \\
\hline - Excessive, much mucus & 0 & $1(3)$ & $1(1)$ \\
\hline • Insufficient, mucinous & $8(21)$ & $4(11)$ & $12(16)$ \\
\hline \multicolumn{4}{|l|}{ Denture plaque } \\
\hline •Clean & $13(34)$ & $7(19)$ & $20(27)$ \\
\hline • Mild debris & $13(34)$ & $20(56)$ & $33(45)$ \\
\hline - Severe debris & $12(32)$ & $9(25)$ & $21(28)$ \\
\hline Upper denture retention & $19(63)$ & $19(61)$ & $38(62)$ \\
\hline Upper lateral displacement & $13(43)$ & $19(61)$ & $32(53)$ \\
\hline \multicolumn{4}{|l|}{ Upper denture resilience } \\
\hline •Firm & $25(64)$ & $25(68)$ & $50(66)$ \\
\hline • Resilient & $9(23)$ & $5(13)$ & $14(18)$ \\
\hline - Flabby & $5(13)$ & 7 (19) & $12(16)$ \\
\hline
\end{tabular}

Missing data: 0-20\% per variable.

\subsection{Sample size}

Based on our pilot study results[14], minimal clinically meaningful inter-group difference in Candida CFU/ml mean change score was considered 30.0 with a standard deviation of 31.6. Considering a dropout rate of $10 \%$ and power of $85 \%$ to reject the null hypothesis, sample size in each group was set to 26 participants. Sample size estimation was calculated by using the Statulator online tool[25].

\subsection{Statistical methods}

Statistical analyses included descriptive analyses and generalized estimating equations, considering follow-up time (paired factor) and the two-factor interaction. The primary outcome variable (CFU counts) underwent log10 (CFU+1) transformation for better data distribution and interpretation. Furthermore, it was tested based on pre- post-treatment differences, to compensate for mild imbalances 
Table 3. Estimates from GEE, pre-post differences in Candida spp. counts (log10 transformed)

\begin{tabular}{|c|c|c|c|c|c|}
\hline \multicolumn{4}{|c|}{ Source } & B & $95 \%$ wald Cl \\
\hline \multirow{8}{*}{ Complete dataset } & \multirow[t]{4}{*}{ Denture biofilm } & Intercept & & 0.2 (SD 0.6) & $(-1.0$ to 1.5$)$ \\
\hline & & Groups & Intervention-Control* & $1.3(\mathrm{SD} 0.8)$ & $(-0.2$ to 2.8$)$ \\
\hline & & Time & 3 months- 6 months* & $-0.2(S D 0.3)$ & $(-0.8$ to 0.4$)$ \\
\hline & & Interaction & Intervention group at 3 months - Other combinations* & $0.2(\mathrm{SD} 0.6)$ & $(-0.9$ to 1.3$)$ \\
\hline & \multirow[t]{4}{*}{ Palatal mucosa } & Intercept & & $0.7(\mathrm{SD} 0.5)$ & $(-0.2$ to 1.7$)$ \\
\hline & & Groups & Intervention-Control* & $-0.3(S D 0.5)$ & $(-1.4$ to 0.8$)$ \\
\hline & & Time & $3 \mathrm{mo}-6 \mathrm{mo}^{*}$ & $-0.2(S D 0.2)$ & $(-0.7$ to 0.3$)$ \\
\hline & & Interaction & Intervention group at 3 months - Other combinations* & $0.3(\mathrm{SD} 0.4)$ & $(-0.5$ to 1.1$)$ \\
\hline \multirow{8}{*}{$\begin{array}{l}\text { Candida-positive } \\
\text { on baseline }\end{array}$} & \multirow[t]{4}{*}{ Denture biofilm } & Intercept & & $0.2(\mathrm{SD}-0.7)$ & $(1.1$ to 0.2$)$ \\
\hline & & Groups & Intervention- Control* & $1.4(S D 0.1)$ & (2.8 to 1.4$)$ \\
\hline & & Time & 3 months -6 months* & $0.5(S D-0.2)$ & (1.3 to 0.5$)$ \\
\hline & & Interaction & Intervention group at 3 months- Other combinations* & $-1.4(S D-2.7)$ & $(-0.1$ to -1.4$)$ \\
\hline & \multirow[t]{4}{*}{ Palatal mucosa } & Intercept & & $0.4(S D 0.3)$ & $(-0.2$ to 1.1$)$ \\
\hline & & Groups & Intervention-Control* & 0.0 (SD 0.3) & $(-0.6$ to 0.6$)$ \\
\hline & & Time & 3 months- 6 months & $0.2(\mathrm{SD} 0.3)$ & $(-0.4$ to 0.8$)$ \\
\hline & & Interaction & Intervention group at 3 months - Other combinations* & $-0.5(S D 0.4)$ & $(-1.3$ to 0.3$)$ \\
\hline
\end{tabular}

* Reference value

Note: reference values were generated in multiple imputations.

Table 4. Effect of brushing on palatal inflammation according to Schwartz index

\begin{tabular}{|c|c|c|c|c|c|c|c|c|c|c|c|c|c|}
\hline \multirow{2}{*}{$\begin{array}{l}\text { Variables } \\
\mathrm{N}(\%) \\
\text { Inflammation area }\end{array}$} & \multicolumn{5}{|c|}{$\begin{array}{c}\text { Palatal Brushing } \\
\mathrm{n}(\%)\end{array}$} & \multirow[t]{2}{*}{ Total } & \multicolumn{5}{|c|}{$\begin{array}{c}\text { Standard Hygiene } \\
\mathrm{n}(\%)\end{array}$} & \multirow[t]{2}{*}{ Total } & \multirow[t]{2}{*}{$p$-value ${ }^{*}$} \\
\hline & 0 & 1 & 2 & 3 & & & 0 & 1 & 2 & 3 & & & \\
\hline Baseline & $0(0)$ & $11(28)$ & $19(48)$ & $10(25)$ & & 40 & $0(0)$ & $13(35)$ & $17(46)$ & $7(19)$ & & 37 & 0.40 \\
\hline 3-month follow-up & $4(11)$ & $21(55)$ & $8(21)$ & $5(13)$ & & 38 & $2(6)$ & $13(38)$ & $11(32)$ & $8(24)$ & & 34 & 0.07 \\
\hline 6-month follow-up & 10(29) & $13(37)$ & $4(11)$ & $8(23)$ & & 35 & $5(16)$ & $10(32)$ & $8(26)$ & $8(26)$ & & 31 & 0.20 \\
\hline Inflammation severity & 0 & 1 & 2 & 3 & & & 0 & 1 & 2 & 3 & & & \\
\hline Baseline & $0(0)$ & $15(38)$ & $18(45)$ & $7(18)$ & & 40 & $0(0)$ & $14(38)$ & $20(54)$ & $3(8)$ & & 37 & 0.60 \\
\hline 3-month follow-up & $4(11)$ & $26(68)$ & $5(13)$ & $3(8)$ & & 38 & $2(6)$ & $18(53)$ & $10(29)$ & $4(12)$ & & 34 & 0.08 \\
\hline 6-month follow-up & 10(29) & $14(41)$ & $8(24)$ & $2(6)$ & & 35 & $5(16)$ & 12(39) & $11(36)$ & $3(10)$ & & 31 & 0.13 \\
\hline Newton classification & 0 & la & lb & $\|$ & III & & 0 & la & $\mathrm{lb}$ & II & III & & \\
\hline Baseline & $0(0)$ & $6(15)$ & $4(10)$ & $23(58)$ & $7(18)$ & 40 & $0(0)$ & $6(16)$ & $6(16)$ & 18(49) & $7(19)$ & 37 & 0.72 \\
\hline 3-month follow-up & $4(11)$ & $6(16)$ & 15(39) & $10(26)$ & $3(8)$ & 38 & $2(6)$ & $6(18)$ & $8(24)$ & $15(44)$ & $3(9)$ & 34 & 0.24 \\
\hline 6-month follow-up & $9(26)$ & $6(17)$ & $7(20)$ & $11(31)$ & $2(6)$ & 34 & $5(16)$ & $5(16)$ & $6(19)$ & $13(42)$ & $2(6)$ & 31 & 0.30 \\
\hline
\end{tabular}

${ }^{*} \mathrm{p}$-values refer to between-group comparisons within each time period (Mann-Whitney test).

Table 5 . Between-group mean differences for each follow-up (and 95\% Cl)

\begin{tabular}{lccc}
\hline Variable & Dataset & 3 months & 6 months \\
\hline Candida, denture & Pooled, $\mathrm{MI}^{*}$ & $0.3(-0.9$ to 1.4$)$ & $0.5(-0.9$ to 1.8$)$ \\
biofilm & Complete cases & $0.3(-0.7$ to 1.4$)$ & $0.7(-0.5$ to 1.8$)$ \\
Candida, palatal & Pooled, $\mathrm{MI}^{*}$ & $-0.3(-1.1$ to 0.5$)$ & $0.0(-0.6$ to 0.7$)$ \\
mucosa & Complete cases & $-0.4(-1.2$ to 0.4$)$ & $0.2(-0.4$ to 0.9$)$ \\
Inflammation & Pooled, $\mathrm{MI}^{*}$ & $-0.7(-1.4$ to 0.1$)$ & $-0.6(-1.5$ to 0.3$)$ \\
& Complete cases & $-0.7(-1.4$ to 0.1$)$ & $-0.6(-1.6$ to 0.3$)$ \\
\hline
\end{tabular}

${ }^{*}$ MI: multiple imputation.

Note: Candida counts were based on log-transformed pre-post treatment differences, and inflammation refers to the total inflammation scores (area + severity) observed at each follow-up. Positive mean differences mean higher values for Group 1 (palatal brushing).

at baseline.

An identity link function and generalized score statistics were used in the generalized estimating equations (GEE), and an ex- changeable working correlation was assumed. Effect estimates for each factor were shown as mean values and unstandardized regression coefficient (B), with respective $95 \%$ confidence intervals. All data analyses were performed on an intention-to-treat basis. The level of statistical significance for all tests was considered as 0.05 . SPSS software (v. 22.0, IBM Corp, v. 22.0. Armonk, NY, USA) was used to conduct all analyses.

Given the considerable number of non-carriers of Candida spp., we conducted a subgroup analysis including just participants who were Candida-positive on baseline. The GEE was redone for the primary outcome as above but considering just participants with $\geq 1$ CFU in at least one of the sites (denture and palate).

Missing data was imputed for Candida spp. count changes and total inflammation score (Schwartz et al. index)[23]. Five datasets were obtained by multiple imputation based on least squares regression, with GEE redone with pooled imputations. 


\section{RESULTS}

The three clinical sites invited 326 patients to participate in this trial (Figure 1 presents the study flowchart). Tables 1 and $\mathbf{2}$ present sociodemographic and clinical characteristics of the study participants. The mean age of the study participants (12 men and 57 women) was 69 years (SD: 11). There was no statistically significant baseline data difference between the two study arms. Participants were completely edentulous for a mean time of 30 (SD: 15) years, with an average current upper denture age of 17 (SD: 15) years. All dentures had acrylic resin bases and teeth. Forty-five percent of participants reported that they did not receive any oral and denture hygiene instructions prior to the study. A smaller number of individuals used a denture cleaner (15\%) compared to mouthwash (36\%), and more than half of the participants (65\%) were wearing their dentures overnight. Also, only $6 \%$ of participants used any adhesive for their upper dentures. Regarding medical history, most participants (48\%) had some systemic disease, the most common being thyroid diseases (16\%) and hypertension (14\%). Most participants (73\%) were using medications, although there was no clear predominant type of medication. Salivary quality was deemed adequate in $83 \%$ of participants.

\subsection{Primary outcome}

Candida spp. were detected in $76 \%$ of study participant biological samples, with $71 \%$ retrieved from denture biofilm (intervention: 73\%; control: $70 \%$ ) and only $39 \%$ from palatal mucosa biofilm (intervention: 30\%; control: 49\%) (Fig. 2). At baseline, there was no significant between-group difference regarding Candida spp. colony forming unit (CFU) counts for denture biofilm samples (Mann-Whitney $U$, $p=0.93)$, however, this difference was statistically significant for the palatal mucosa $(p=0.02)$.

- Denture Biofilm: Pre-post intervention changes (positive values mean favourable results/reduction after treatment; whereas negative results represent that post-intervention counts were higher than baseline) in Candida spp. log10-transformed counts $(95 \% \mathrm{Cl})$ were 0.0 (-0.6 to 0.6$)$ at 3-month follow-up, and 0.5 (-0.2 to 1.3) for the control group. At 6-month follow-up, pre-post intervention difference in transformed counts were $0.6(95 \% \mathrm{Cl}:-0.2$ to 1.4$)$ and $-0.1(95 \%$ $\mathrm{Cl}$ : -0.9 to 0.7$)$ for intervention and control, respectively. Although group and time exerted no significant effect on those counts (GEE - complete dataset, $p=0.85$ and 0.10 , respectively), the interaction between the group and the time was significant $(p=0.02)$. Parameter estimates were not significant (Table 3).

- Palatal mucosa: Pre-post treatment change scores in Candida spp. log10-transformed counts were $-0.3(95 \% \mathrm{Cl}:-0.7$ to 0.2$)$ for intervention group at 3 months, and 0.2 (95\% Cl: -0.3 to 0.7$)$ for the control group. At 6 months, transformed counts changed to 0.1 (95\% $\mathrm{Cl}$ - -0.2 to 0.4$)$ in the intervention group and $-0.1(95 \% \mathrm{Cl}:-0.7$ to 0.4$)$ in the control group. Both group and time exerted no significant effect on those counts (GEE - complete dataset, $\mathrm{p}=0.69$ and 0.78 , respectively), as well as their interaction $(p=0.09)$. Parameter estimates were not significant (Table 3).

- Research centres: For denture biofilm and palatal mucosa, centres had no significant effect (GEE, $p=0.24$ and 0.05 , respectively). The interaction between groups and centres was not significant either ( $p=0.22$ for denture biofilm and 0.43 for palatal mucosa).
- Subgroup analysis: For the intervention group, the number of Candida-positive participants who concluded the 3- and 6-month follow-ups were 27 and 24, respectively; 27 control group participants concluded both 3- and 6-month follow-ups (Table 3). For the log-transformed Candida count changes in denture biofilm, the group-time interaction was significant ( $G E E, p=0.04)$, but not for each factor separately ( $p$, group $=0.21$; time $=0.67$ ). Groups and time yielded more distinct results evident with subgroup analysis, with estimated means being: 3 months, intervention: 0.8 (95\% Cl: 0.0 to 1.5) and control: 0.7 (95\% Cl: -0.2 to 1.7$) ; 6$ months, intervention: $1.6(95 \%$ Cl: 0.7 to 2.6) and control: 0.2 (-0.7 to 1.1). Changes in Candida counts from the palatal mucosa were not affected by group ( $G E E, p=0.46)$, time $(p=0.86)$, or their interaction $(p=0.27)$. Log-transformed differences at 3 months were: intervention: $0.1(95 \% \mathrm{Cl}:-0.5$ to 0.8$)$ and control: 3 months: 0.7 (95\% Cl: -0.1 to 1.4); at 6 months, intervention: 0.4 ( $95 \% \mathrm{Cl}: 0.1$ to 0.8 ) and control: 0.4 ( $95 \% \mathrm{Cl}:-0.2$ to 1.1$)$.

\subsection{Secondary outcome}

Table 4 presents data on the effect of palatal brushing on inflammation by the Schwartz index. Regardless of the group, almost half of the participants had inflammation affecting $25-50 \%$ of the denture-bearing area, and nearly one-quarter had either $<25 \%$ or $>50 \%$ in area. Severity of inflammation tended to be moderate in nearly half of the cases, but more participants presented mild rather than severe inflammation. There were no significant between-group differences for total inflammation scores (Mann-Whitney $\mathrm{U}, \mathrm{p}=0.436$ ), as well as the area $(p=0.409)$ and severity $(p=0.609)$ at baseline. As for the Newton classification, most participants presented stomatitis class II, whereas almost one-fifth had class III, and between 10 to $16 \%$ had some of the class I subdivisions.

Figure 3 provides data on palatal inflammation, showing improvement over time and slightly lower scores with palatal brushing. Although time $(G E E, p<0.001)$ and the group-time interaction $(p=0.01)$ were significant, groups had no significant effect $(p=0.50)$. Estimated mean scores after 3 months were 3.8 ( $95 \% \mathrm{Cl}: 3.4$ to 4.2 ) for the intervention group and 3.5 ( $95 \% \mathrm{Cl}$ : 3.1 to 3.9) for the control group; at 6 months, scores dropped to 2.6 (95\% Cl: 2.1 to 3.0) for the intervention and 3.2 (95\% Cl: 2.7 to 3.7$)$ for the control. Post hoc comparisons showed lower scores for the intervention at 6 months compared to both groups at 3 months, whereas intermediate scores were found for the control at 6 months. Transforming 6-month scores into treatment effect (Budtz-Jorgensen Index) showed better improvement with palatal brushing compared to solely hygiene instructions (Fig. 3). It showed less inflammation for $70 \%$ and $45 \%$ of the intervention and control participants, respectively (significant, chi-square, $\mathrm{p}=0.04)$. Those proportions represented a number needed to treat (NNT) of 3.9 (95\% Cl: 2.1 to 44.4 ).

\subsection{Multiple imputation}

Compared to the primary data, pooled imputed data reached close results for all tested outcome variables, i.e., Candida counts in denture and palatal biofilm, and total inflammation scores. Both mean differences between groups and their respective $95 \% \mathrm{Cl}$ were similar for both pooled data and complete cases (Table 5).

\subsection{Adverse effects}

Most participants were experiencing no pain at each time point, and between-group differences were not significant. At baseline, 


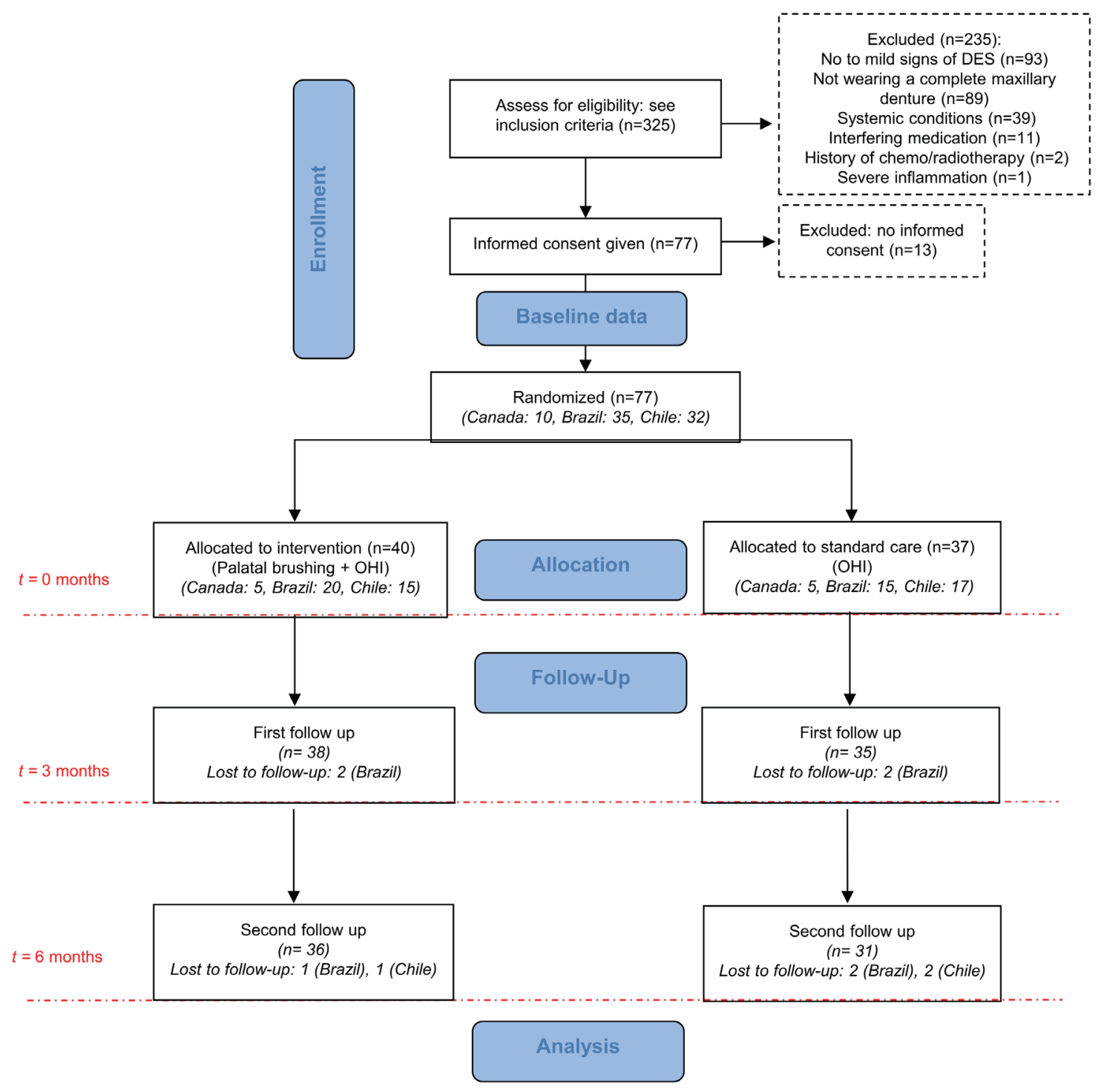

Fig. 1. CONSORT study flowchart, with separate numbers for each clinical site from baseline to 6 months.

mild pain was never experienced by 25 (63\%) intervention and 26 (70\%) control group participants (Mann-Whitney, $p=0.674$ ). After oral hygiene instructions, participants without mild pain corresponded to: $30(79 \%)$ intervention and $26(74 \%)$ control at 3 months $(p=0.796)$; $28(78 \%)$ intervention and $23(74 \%)$ control at 6 months $(p=0.687)$. No participant reported any adverse effect that led them to stop palatal brushing or any of the standard oral hygiene methods, including moderate to severe pain or persistent mucosal bleeding.

\section{DISCUSSION}

To our best knowledge, this is the first randomized trial that has examined the role of palatal brushing in the treatment of DES. This trial provides evidence in favour of adding palatal brushing to the hygiene instructions of denture wearing patients. No statistically significant change in Candida carriage on the palatal mucosa was found; however, it is well known that denture biofilm, and not palatal biofilm, is the primary harbouring site for Candida. As for inflammation, a significant decrease was found in both groups in the denture- bearing mucosa, with larger effect in the palatal brushing group. These results are in line with the current literature on the efficacy of palatal brushing and oral hygiene methods in controlling DES[14].

The decrease in Candida carriage on upper dentures shows how palatal brushing can treat DES. This finding discloses that mucosal hygiene affects the composition of denture biofilm, most likely due to the dissipation of by-products of tissue injury/inflammation. Denture biofilm can harbour pathogenic bacteria and yeasts, which can produce toxins and metabolic wastes and thus inflammation of the mucosa[1,14]. A vicious circle takes place, since the inflamed mucosa produces proteins that stimulate candidal growth and virulence[1,26]. Thus, palatal brushing complements standard hygiene methods in breaking that circle, with the first reducing inflammation by-products while the latter removes denture biofilm mechanically.

The relevance of salivary composition and quantity in the results of palatal brushing deserves further comments. Part of the effect of palatal brushing may be explained by the mechanical stimulation of 


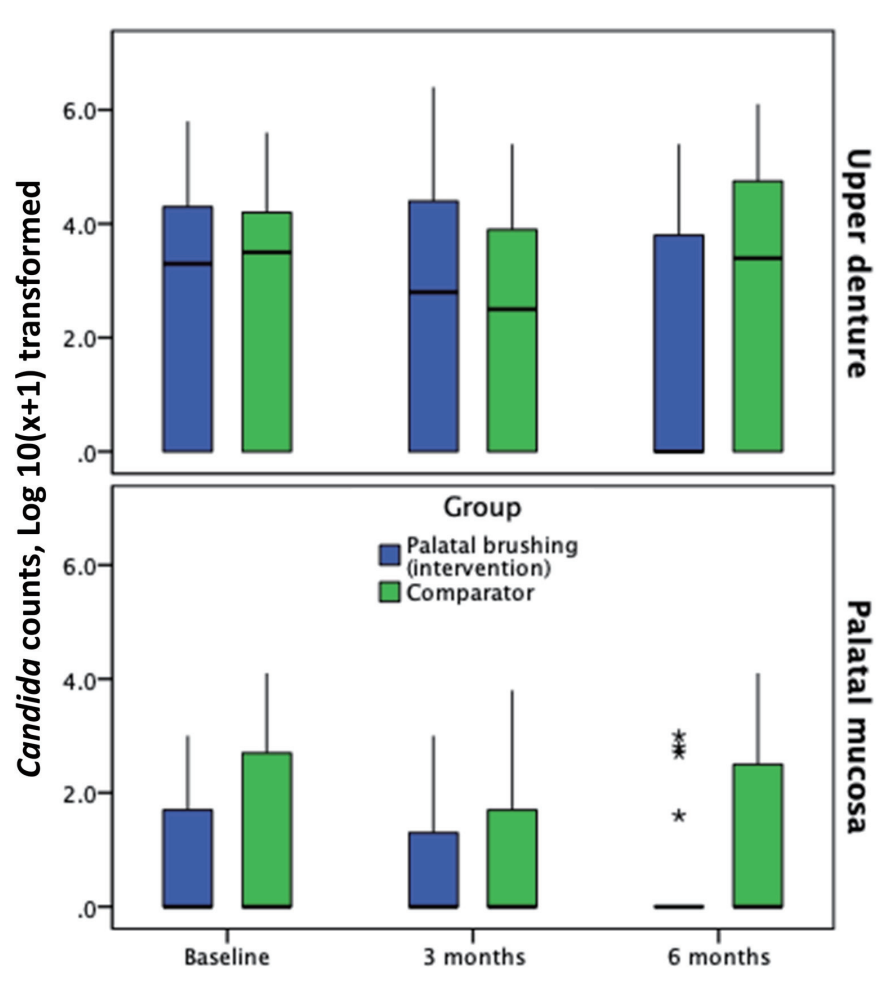

Fig. 2. Box-plot graph with Candida spp. counts before and after treatment according to group allocation and site.

minor palatal salivary glands. An increased salivary flow from those glands may be behind at least part of the overall positive effect of palatal brushing[15]. Indeed, xerostomia is a well-known predisposing factor in the development of DES and Candida infection[27-29]. Both proper salivary flow and saliva composition can reduce the risk of DES and Candida carriage by offering mechanical cleansing of the denture biofilm and transporting immune factors specific to fungal microorganisms such as lactoferrin, histatin-5, and many others[14,30-33]. Therefore, palatal brushing most likely acts by stimulating the self-cleansing of the oral cavity, besides the mechanical/ direct elimination of inflammation by-products.

When Candida was counted directly from the palatal mucosa instead of denture biofilm, post-treatment results were not different for both trial arms. This is not surprising since denture biofilm tends to harbour a larger and more diverse yeast population[33-36]. More precisely, unlike living mucosa, the abiotic surface of a denture does not have a self-renewal mechanism that ensures the elimination of biofilms[35-37]. Furthermore, all dentures evaluated were made of acrylic resin, which can be deteriorated over the years by enzymes and metabolites from biofilm[38]. Thus, the ageing of denture base materials has certainly led to increased roughness and susceptibility to Candida adhesion[34,36,37]. Therefore, it is not surprising that Candida carriage was significantly reduced in the denture and not the palate, but still induced an improvement in clinical signs of DES and inflammation.

It is important to highlight that more than half of the participants wore their dentures overnight during our clinical study, which can have affected our results for oral inflammation and Candida count[39]. However, we have not included instructions to avoid over- night denture wearing in this trial to maintain a pragmatic framework, i.e., to observe the effect of palatal brushing over "real-life" conditions. It is important to highlight that the rate of overnight wearing was evenly distributed amongst the two study groups on baseline, thus unlikely able to influence post-intervention betweengroup differences. The same can be said for all sociodemographic and clinical characteristics of participants, including denture retention, the use of denture cleaners and mouthwash.

The significant reduction in inflammation area and severity found at the 6-month follow-up also supports the efficacy of palatal brushing in the care management of DES. Indeed, mechanical stimulation has been demonstrated to stimulate keratinization, reduce inflammatory infiltrates, increase the proliferation of fibroblast and collagen synthesis, improve microcirculation, and as already mentioned, the salivary flow[26,27,29,31]. Through these mechanisms, palatal brushing can stimulate mucosal repair and eliminate the inflammatory processes underlying the development of DES. Moreover, the decrease in inflammation can also partly explain the decrease in Candida carriage that was observed. Indeed, as the inflammatory state decreased, the factors produced by the inflamed mucosa that stimulates the development of Candida can also decrease, distinguishing palatal brushing from other hygiene methods that solely address the denture biofilm[26]. Supporting this hypothesis, even if standard hygiene instructions did improve the clinical signs of DES in the comparison group, the effect did not reach the extent of improvement seen in the palatal brushing group.

From a clinical perspective, palatal brushing has demonstrated a net superiority in reducing clinical signs of DES. Not only did this method lead to significant improvement in most participants, it also established a number needed to treat of less than four patients. In other words, a clinician recommending palatal brushing to address DES will need to treat four patients to see an improvement in one of them, compared to standard hygiene instructions. It is worth mentioning that most studies using antifungals have reported rapid recurrence of the conditions, some as soon as 14 days after the discontinuation of treatment $[8,40]$. In contrast, palatal brushing maintained a linear trajectory of decrease in clinical signs of DES, inflammation, and Candida carriage. Further investigations need to be carried out to examine long-term outcome of palatal brushing.

The authors acknowledge that this trial has limitations. The multicentre design of this study might have resulted in discrepancies among study participants from different centres. However, subjects were compared at baseline to ensure comparability of the study groups, and the statistical analysis did not find any centre influence on the outcomes. In reality, a multicentre design could enable a larger sample and enhance the generalizability of results to different populations. This result is in line with the phase one trial findings demonstrating no effect of recruiting centre on baseline data and intervention outcomes[14]. However, contrary to the phase one trial, some participants were lost to follow-up. To address this limitation, an imputation strategy showed that incomplete data has not led to biased results.

The pragmatic approach of this trial and its intention-to-treat analysis also ensure that its results can still be reproduced in the general population. The results obtained in this study can be hypothesized as being as close as possible to a real-life setting where clinicians cannot ensure their patient's compliance with treatment. 

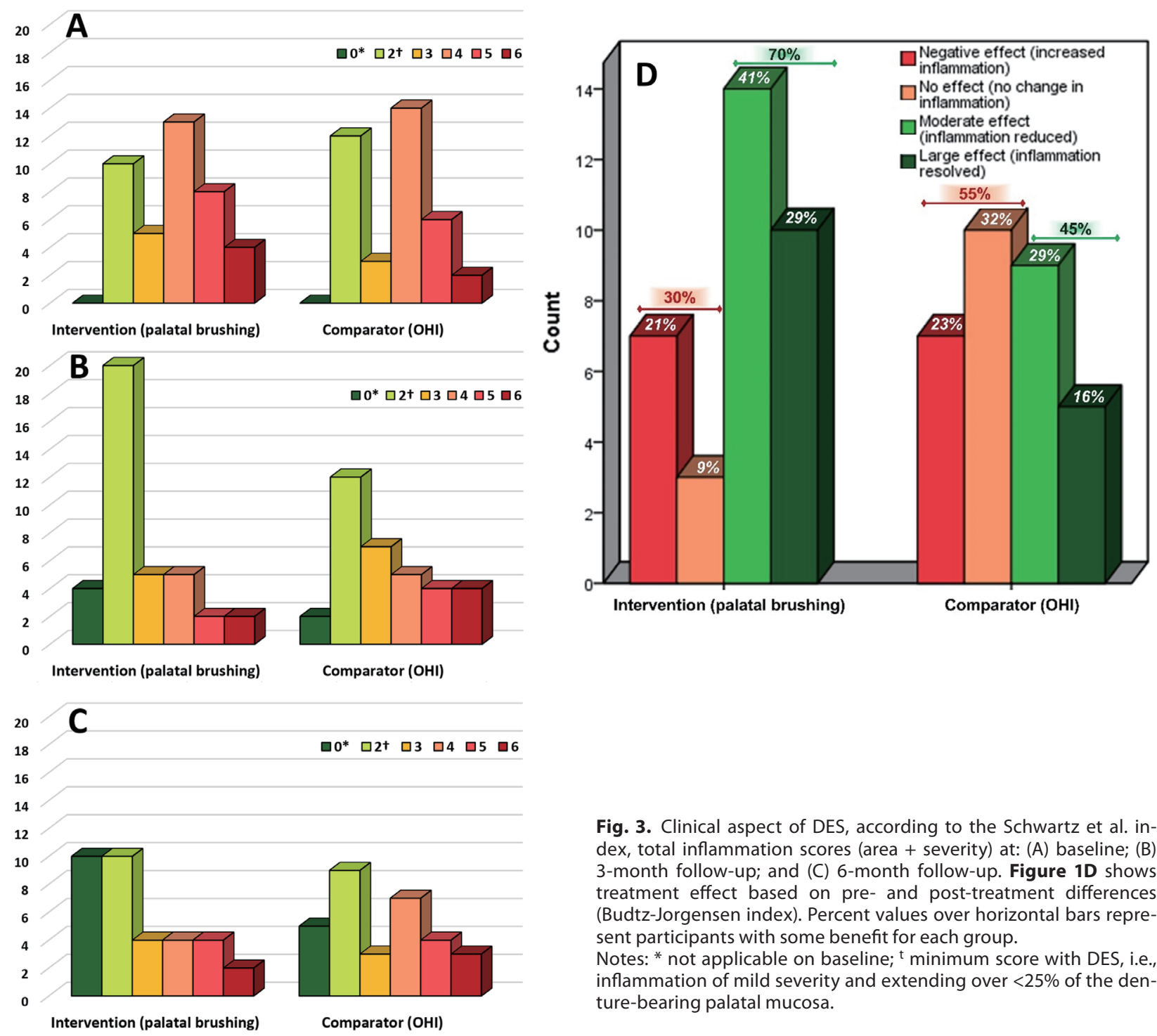

Fig. 3. Clinical aspect of DES, according to the Schwartz et al. index, total inflammation scores (area + severity) at: (A) baseline; (B) 3-month follow-up; and (C) 6-month follow-up. Figure 1D shows treatment effect based on pre- and post-treatment differences (Budtz-Jorgensen index). Percent values over horizontal bars represent participants with some benefit for each group.

Notes: ${ }^{*}$ not applicable on baseline; ${ }^{\mathrm{t}}$ minimum score with DES, i.e., inflammation of mild severity and extending over $<25 \%$ of the denture-bearing palatal mucosa.

Another noteworthy aspect refers to the finding of lower Candida counts with the intervention group, which was statistically significant only in subgroup analysis. This is not surprising, since less effect could be expected when the data are diluted with Candidanegative participants. Even though this kind of subgroup analysis often deserves a conservative interpretation, results are strengthened by their consistency with primary analysis and the study findings on the effect of palatal brushing on mucosal inflammation[41].

In summary, this study supports the result of the previous trial and the effectiveness of palatal brushing in DES treatment. The authors recommend using this simple and cost-effective new therapy, along with standard hygiene instructions, to treat DES.

\section{Acknowledgments}

This study was supported by funds from the CIHR Phase II Clinician Scientist Award (award \#201110, E. Emami), FRQ-S RSBO Recruitment Aid (grant BRDV-FRQS-201-2016-046, RF de Souza), and internal grant by Universidad de la Frontera (grant DIUFRO-DIE15-0003, E. Bo-

rie). RF de Souza is the recipient of a "Clinical Research Scholar" salary award from the FRQ-S (dossier 252635). The authors thank Pierre Rompré for his assistance with analytical planning.

\section{Conflicts of interest}

The authors have no known competing financial interests or personal relationships that could have appeared to influence the work reported in this paper.

\section{References}

[1] Gendreau L, Loewy ZG. Epidemiology and etiology of denture stomatitis. J Prosthodont. 2011;20:251-60. https://doi.org/10.1111/j.1532849X.2011.00698.x, PMID:21463383

[2] Hilgert JB, Giordani JMA, de Souza RF, Wendland EMDR, D'Avila OP, Hugo FN. Interventions for the Management of Denture Stomatitis: A Systematic Review and Meta-Analysis. J Am Geriatr Soc. 2016;64:2539-45. https://doi. org/10.1111/jgs.14399, PMID:27889906 
[3] Rocha Gusmão JM, Ferreira dos Santos SS, Piero Neisser M, Cardoso Jorge AO, Ivan Faria M. Correlation between factors associated with the removable partial dentures use and Candida spp. in saliva. Gerodontology. 2011;28:283-8. https://doi.org/10.1111/j.1741-2358.2010.00390.x, PMID:20662943

[4] Mattos BSC, Sousa AA, Magalhães MHCG, André M, Brito e Dias R. Candida albicans in patients with oronasal communication and obturator prostheses. Braz Dent J. 2009;20:336-40. https://doi.org/10.1590/S010364402009000400013, PMID:20069259

[5] Shimizu K, Shimizu F, Kamiyama K. Microbiological studies on dentureinduced stomatitis in children. Pediatr Dent. 1987;9:304-7. PMID:3507649

[6] Osmenda G, Maciąg J, Wilk G, Maciąg A, Nowakowski D, Loster J, et al. Treatment of denture-related stomatitis improves endothelial function assessed by flow-mediated vascular dilation. Arch Med Sci. 2017;1:66-74. https://doi. org/10.5114/aoms.2017.64715, PMID:28144257

[7] Fedele S, Sabbah W, Donos N, Porter S, D'Aiuto F. Common oral mucosal diseases, systemic inflammation, and cardiovascular diseases in a large crosssectional US survey. Am Heart J. 2011;161:344-50. https://doi.org/10.1016/j. ahj.2010.11.009, PMID:21315218

[8] Emami E, Kabawat M, Rompre PH, Feine JS. Linking evidence to treatment for denture stomatitis: A meta-analysis of randomized controlled trials. J Dent. 2014;42:99-106. https://doi.org/10.1016/j.jdent.2013.11.021, PMID:24316341

[9] Hannah VE, O'Donnell L, Robertson D, Ramage G. Denture Stomatitis: Causes, Cures and Prevention. Prim Dent J. 2017;6:46-51. https://doi. org/10.1308/205016817822230175, PMID:29258641

[10] Emami E, de Grandmont P, Rompré PH, Barbeau J, Pan S, Feine JS. Favoring trauma as an etiological factor in denture stomatitis. J Dent Res. 2008;87:440-4. https://doi.org/10.1177/154405910808700505, PMID:18434573

[11] Yarborough A, Cooper L, Duqum I, Mendonça G, McGraw K, Stoner L. Evidence Regarding the Treatment of Denture Stomatitis. J Prosthodont. 2016;25:288-301. https://doi.org/10.1111/jopr.12454, PMID:27062660

[12] Araujo CB, Ribeiro AB, Fortes CV, Bueno FL, De Wever B, Oliveira VC, et al. Effect of local hygiene protocols on denture-related stomatitis, biofilm, microbial load, and odor: A randomized controlled trial. J Prosthet Dent. 2021;S0022-3913(20)30808-8. https://doi.org/10.1016/j.prosdent.2020.12.018, PMID:33736863

[13] Badaró MM, Bueno FL, Arnez RM, Oliveira VC, Macedo AP, de Souza RF, et al. The effects of three disinfection protocols on Candida spp., denture stomatitis, and biofilm: A parallel group randomized controlled trial. J Prosthet Dent. 2020;124:690-8. https://doi.org/10.1016/j.prosdent.2019.09.024, PMID:31955835

[14] Kabawat $M$, Souza R, Badaró $M$, Koninck L, Barbeau J, Rompré $P$, et al. Phase 1 clinical trial on the effect of palatal brushing on denture stomatitis. Int J Prosthodont. 2014;27:311-9. https://doi.org/10.11607/ijp.3844, PMID:25010873

[15] de Souza RF, Khiyani MF, Chaves CAL, Feine J, Barbeau J, Fuentes R, et al. Improving practice guidelines for the treatment of denture-related erythematous stomatitis: a study protocol for a randomized controlled trial. Trials. 2017;18:211. https://doi.org/10.1186/s13063-017-1947-y, PMID:28476133

[16] Emami E, Séguin J, Rompré PH, de Koninck L, de Grandmont $P$, Barbeau J. The relationship of myceliated colonies of Candida albicans with denture stomatitis: an in vivo/in vitro study. Int J Prosthodont. 2007;20:514-20. PMID:17944343

[17] Webb BC, Thomas CJ, Whittle T. A 2-year study of Candida-associated denture stomatitis treatment in aged care subjects. Gerodontology. 2005;22:168-76. https://doi.org/10.1111/j.1741-2358.2005.00065.x, PMID:16163908

[18] Silva M, Consani R, Sardi J, Mesquita M, Macêdo A, Takahashi J. Microwave irradiation as an alternative method for disinfection of denture base acrylic resins. Minerva Stomatol. 2013;62:23-9. PMID:23422681

[19] Brooks JF, Hooper LV, eds. Interactions among microbes, the immune system, and the circadian clock. Seminars in Immunopathology; 2020: Springer.

[20] Williamson JJ. Diurnal variation of Candida albicans counts in saliva. Aust Dent J. 1972;17:54-60. https://doi.org/10.1111/j.1834-7819.1972.tb02746.x, PMID:4557268
[21] Salles MM, Badaró MM, Arruda CNF, Leite VMF, Silva CHL, Watanabe E, et al. Antimicrobial activity of complete denture cleanser solutions based on sodium hypochlorite and Ricinus communis - a randomized clinical study.J Appl Oral Sci. 2015;23:637-42. https://doi.org/10.1590/1678-775720150204, PMID:26814466

[22] Newton A.Denture sore mouth:A possible aetiology. BrDent J. 1962;112:357.

[23] Schwartz IS, Young JM, Berrong JM. The effect of Listerine antiseptic on denture microbial flora and denture stomatitis. Int J Prosthodont. 1988;1:153-8. PMID:3250571

[24] Budtz-Jörgensen E, Löe H. Chlorhexidine as a denture disinfectant in the treatment of denture stomatitis. Eur J Oral Sci. 1972;80:457-64. https://doi. org/10.1111/j.1600-0722.1972.tb00314.x, PMID:4575037

[25] Dhand NK, Khatkar MS. Statulator: An online statistical calculator. Sample Size Calculator for Comparing Two Independent Means 2014 [Available from: http://statulator.com/SampleSize/ss2M.html.

[26] Custodio W, Silva WJ, Paes Leme AF, Cury JA, Del Bel Cury AA. Plasma proteins in the acquired denture pellicle enhance substrate surface free energy and Candida albicans phospholipase and proteinase activities. J Investig Clin Dent. 2015;6:273-81. https://doi.org/10.1111/jicd.12101, PMID:25045148

[27] Pereira-Cenci T, Del Bel Cury AA, Crielaard W, Ten Cate JM. Development of Candida-associated denture stomatitis: new insights. J Appl Oral Sci. 2008;16:86-94. https://doi.org/10.1590/S1678-77572008000200002, PMID:19089197

[28] Nakagawa Y. Influence of dry mouth and denture use as predisposing factors for erythematous candidiasis. Annals of Japan Prosthodontic Society. 2018;10:32-9. https://doi.org/10.2186/ajps.10.32

[29] Campisi G, Panzarella V, Matranga D, Calvino F, Pizzo G, Lo Muzio L, et al. Risk factors of oral candidosis: A twofold approach of study by fuzzy logic and traditional statistic. Arch Oral Biol. 2008;53:388-97. https://doi.org/10.1016/j. archoralbio.2007.11.009, PMID:18191810

[30] Altarawneh S, Bencharit S, Mendoza L, Curran A, Barrow D, Barros S, et al. Clinical and histological findings of denture stomatitis as related to intraoral colonization patterns of Candida albicans, salivary flow, and dry mouth. J Prosthodont. 2013;22:13-22. https://doi.org/10.1111/j.1532849X.2012.00906.x, PMID:23107189

[31] Walker DM. Oral mucosal immunology: an overview. Ann Acad Med Singap. 2004;33(suppl):27-30. PMID:15389303

[32] Mese H, Matsuo R. Salivary secretion, taste and hyposalivation. J Oral Rehabil. 2007;34:711-23. https://doi.org/10.1111/j.1365-2842.2007.01794.x, PMID:17824883

[33] Williams D, Lewis M. Pathogenesis and treatment of oral candidosis. J Oral Microbiol. 2011;3:5771. https://doi.org/10.3402/jom.v3i0.5771, PMID:21547018

[34] Hahnel S, Rosentritt M, Bürgers R, Handel G, Lang R. Candida albicans biofilm formation on soft denture liners and efficacy of cleaning protocols. Gerodontology. 2012;29:e383-91. https://doi.org/10.1111/j.17412358.2011.00485.x, PMID:21585518

[35] Offenbacher S, Barros SP, Altarawneh S, Beck JD, Loewy ZG. Impact of tooth loss on oral and systemic health. Gen Dent. 2012;60:494-500. PMID:23220304

[36] Williams DW, Kuriyama T, Silva S, Malic S, Lewis MAO. Candida biofilms and oral candidosis: treatment and prevention. Periodontol 2000. 2011;55:25065. https://doi.org/10.1111/j.1600-0757.2009.00338.x, PMID:21134239

[37] Gleiznys A, Zdanavičienè E, Žilinskas J. Candida albicans importance to denture wearers. A literature review. Stomatologija. 2015;17:54-66. PMID:26879270

[38] Matsuo H, Suenaga H, Takahashi M, Suzuki O, Sasaki K, Takahashi N. Deterioration of polymethyl methacrylate dentures in the oral cavity. Dent Mater J. 2015;34:234-9. https://doi.org/10.4012/dmj.2014-089, PMID:25740307

[39] linuma T, Arai Y, Abe Y, Takayama M, Fukumoto M, Fukui Y, etal. Denture wearing during sleep doubles the risk of pneumonia in the very elderly. J Dent Res. 2015;94(suppl):28S-36S. https://doi.org/10.1177/0022034514552493, PMID:25294364

[40] Olsen I. Denture stomatitis: the clinical effects of chlorhexidine and amphotericin B. Acta Odontol Scand. 1975;33:47-52. https://doi. org/10.3109/00016357509004626, PMID:773086

[41] Friedman LM, Furberg C, DeMets DL. Fundamentals of clinical trials. 4th ed. New York: Springer; 2010. xviii, 445 p. p. 
Supplementary Table 1. Assessment of clinical variables

A. Modified Newton classification

0 : Healthy mucosa/no DES.

Type IA: Petechiae in normal palatal tissue, usually found around the orifices of the ducts of the palatal mucous glands

Type IB: Localized area of inflammation of the denture-bearing area

Type II: Generalized area of inflammation of the denture-bearing area

Type III: Hyperplastic palatal surface with inflammation of the denture-bearing area

B. Schwartz et al. inflammation index

B.1. Area

0 : No inflammation

1: Inflammation of the palate extending up to $25 \%$ of the palatal denture-bearing tissue

2: Inflammation of the palate covering between $25 \%$ and $50 \%$ of the palatal denture-bearing tissue

3: Inflammation covering more than $50 \%$ of the palatal denture-bearing tissue

B.2. Severity

0 : Normal tissue

1: Mild inflammation (slight redness, no swelling or edema)

2: Moderate inflammation (redness with some edema)

3: Severe inflammation (acutely inflamed redness, edema)

C. Budtz-Jorgensen Index

Treatment effect based on total inflammation score by the Schwartz et al. index

Large effect: inflammation resolved

B. $1+$ B. 2 at 6 months $=$ zero

Moderate: effect inflammation reduced

B. $1+$ B. 2 at 6 months $<$ B. $1+$ B. 2 at baseline, but $>$ zero

No effect: no change in inflammation

B. $1+$ B. 2 at 6 months = B.1+B.2 at baseline

Negative effect: increased inflammation

B.1+B.2 at 6 months $>$ B. $1+$ B. 2 at baseline 